\title{
Ungesunde Abgase
}

traßenverkehr hat auch bei kurzzeitiger Exposition Auswirkungen auf die Gesundheit - zumindest bei Allergikern. Um dies zu zeigen, setzten sich 26 Patienten mit atopischen Ekzem sowie 26 gesunde Kontrollpersonen für 30 Minuten neben eine vielbefahrene Straße in Kyoto, Japan. Direkt zuvor und danach wurden Pricktests und Blutabnahmen durchgeführt. Während sich bei den Gesunden keine Veränderung zeigte, konnte bei den Ekzempatienten

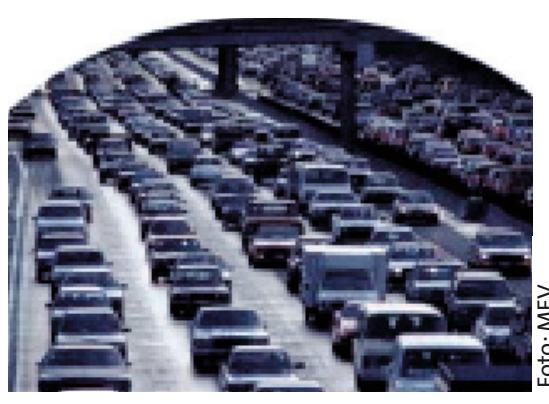

eine Zunahme der Quaddelgröße dokumentiert werden. Dies ist nicht als unspezifische Hautirritation zu werten, denn die Reaktion auf Histamin blieb unverändert.

Kimata H. Int J Hyg Environ Health 2004; 207: 45-9

\section{Weniger Asthma in Australien}

D as kleine Städtchen Belmont in der Nähe von Sydney dient als eine Art Referenz für die Prävalenz atopischer Erkrankungen in Australien. Erstmals waren dort 1982 die Daten aller Schulkinder zwischen 8 und 11 Jahren erhoben worden. Bei der nächsten Auswertung im Jahr 1992 hatte die Asthmaprävalenz dramatisch von $9,1 \%$ auf $38,8 \%$ zugenommen. Bei der aktuellen Erhebung im Jahr 2002 erlebten die Epidemiologen jetzt eine kleine Überraschung: Der Anteil der asthmakranken Grundschüler hatte sich - entgegen dem weltweiten Trend - signifikant reduziert auf $31,0 \%$. Die Zahl der Kinder mit Heuschnupfen und atopischem Ekzem war dagegen in etwa gleich geblieben. Wie verlässlich dieser neue Trend ist, bleibt abzuwarten. ib

Toelle BG et al. BMJ 2004; 328: 386-7

\section{Mehr Kinder - weniger Allergien}

chwangerschaften können Allergien heilen. $\mathrm{Zu}$ diesem verblüffenden Schluss kommt eine englische Arbeitsgruppe, die bei einer Kohorte von 483

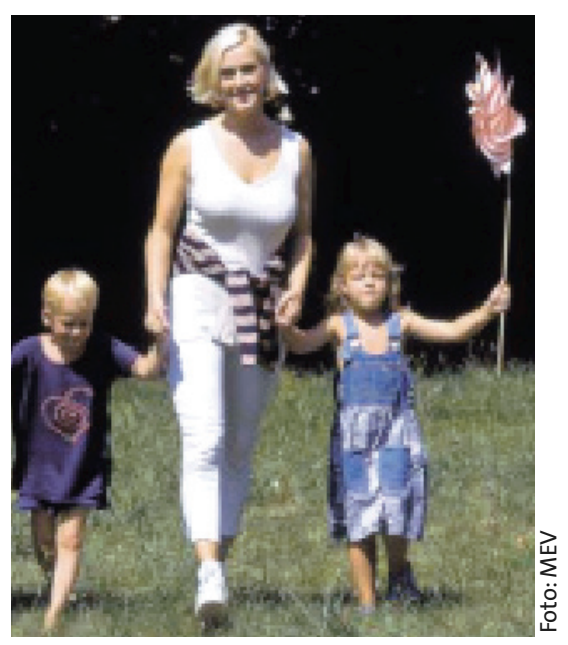

Frauen zweimal im Abstand von durchschnittlich 7 Jahren einen Pricktest mit drei gängigen Allergenen durchführte. Bei 15\% der Frauen, bei denen der Test initial positiv gewesen war, zeigte sich bei der folgenden Testung keine Reaktion mehr. Ein Sistieren von Heuschnupfensymptomen gaben 33\% der Frauen an. Dabei war die Wahrscheinlichkeit für das Abklingen von Allergien umso höher, je öfter die Frauen in der Zwischenzeit entbunden hatten ( $p=0,05$ bzw. $\mathrm{p}=0,02)$. Unter der Annahme, dass der Atopiestatus der Mutter sich auch auf die Kinder überträgt, könnte diese Beobachtung eine Erklärung dafür sein, warum nachgeborene Kinder seltener an Allergien erkranken.

ib

Harris JM et al. Clin Exp Allergy 2004; 34: 369-72

\section{Allergierisiko Hochhaus}

Dollenallergien sind in Städten häufiger als auf dem Land. Doch nicht nur der Wohnort, sondern auch die Wohnhöhe spielt eine Rolle: Wer in höheren Stockwerken lebt, ist häufiger betroffen als Erdgeschoßbewohner. Zu dieser Erkenntnis gelangten spanische Wissenschaftler, die die allergischen Symptome von über 17.000 Stadt- und Landbewohnern dokumentierten und mit der Etagenhöhe ihrer Wohnungen verglichen. Das relative Risiko einer Sensibilisierung gegen Graspollen stieg sogar linear mit der Stockwerkshöhe an $(\mathrm{p}<0,00001)$. Die Autoren vermuten, dass tatsächlich die Pollenbelastung in der Höhe größer ist und raten Allergikern zu einem Umzug in tiefere Stockwerke.

ib

Armentia A et al. Allergy 2004; 59: 302-5

\section{Falsche Hoffnung Fisch}

G enuss von Fisch soll, wohl wegen der in Fischöl enthaltenen mehrfach ungesättigten Omega-3-Fettsäuren, vor entzündlichen Atemwegserkrankungen schützen. Um einem möglichen Zusammenhang zwischen Fettsäuren im Blut und Asthma auf die Spur zu kommen, unterzogen sich über 1.000 Australier Pricktests, Lungenfunktionsprüfungen sowie Blutabnahmen und füllten Fragebögen aus. Das unerwartete Ergebnis: Zwar konnte kein Zusammenhang zwischen im Blut vorhandenen Omega-3-Fettsäuren und Asthma festgestellt werden und auch der Atopiestatus war nicht von den Fettsäuren beeinflusst, dagegen waren jedoch die mehrfach ungesättigten Omega-6-Fettsäuren, insbesondere die Dihomogammalinolensäure (DHGLA), bei Personen mit Asthma signifikant erhöht (OR 1,30, 95\%-KI 1,06-1,60). Der Grund dafür ist laut den Wissenschaftlern noch unklar. Zur Klärung sind weitere Untersuchungen nötig.

Woods RK et al. Thorax 2004; 59: 105-10 